\title{
Building footprint extraction from Digital Surface Models using Neural Networks
}

\author{
Ksenia Davydova*, Shiyong Cui, and Peter Reinartz \\ German Aerospace Center (DLR), Remote Sensing Technology Institute (IMF), \\ Oberpfaffenhofen, Germany
}

\begin{abstract}
Two-dimensional building footprints are a basis for many applications: from cartography to three-dimensional building models generation. Although, many methodologies have been proposed for building footprint extraction, this topic remains an open research area. Neural networks are able to model the complex relationships between the multivariate input vector and the target vector. Based on these abilities we propose a methodology using neural networks and Markov Random Fields (MRF) for automatic building footprint extraction from normalized Digital Surface Model (nDSM) and satellite images within urban areas. The proposed approach has mainly two steps. In the first step, the unary terms are learned for the MRF energy function by a four-layer neural network. The neural network is learned on a large set of patches consisting of both nDSM and Normalized Difference Vegetation Index (NDVI). Then prediction is performed to calculate the unary terms that are used in the MRF. In the second step, the energy function is minimized using a maxflow algorithm, which leads to a binary building mask. The building extraction results are compared with available ground truth. The comparison illustrates the efficiency of the proposed algorithm which can extract approximately $80 \%$ of buildings from nDSM with high accuracy.
\end{abstract}

Keywords: Building footprint extraction, binary mask, Digital Surface Model, neural networks, Markov Random Fields, Normalized Difference Vegetation Index

\section{INTRODUCTION}

Two-dimensional (2D) building footprint extraction from imagery or/and DSM data has been a research issue for decades and is of great interest since it plays a key role in three-dimensional (3D) building model generation, map updating, urban planning and reconstruction, infrastructure development, etc. The collection of building footprints often needs a lot of manual work and is both time consuming and costly. Moreover, it is challenging to extract building information from remotely sensed data due to the sophisticated nature of urban environments. Therefore, automatic methods are required for an efficient collection of building footprints from large urban areas containing thousands of buildings.

Many automatic methods have been developed in the last decades using various data sources. Some approaches perform building detection on the basis of aerial ${ }^{1,2}$ and high-resolution satellite imagery ${ }^{3-7}$ using spectral reflectance values. ${ }^{8}$ However, these methods often face problems caused by imaging radiometry of optical sensors when similar spectral reflectance of the ground occurs. The building roofs are built from different materials depending on the regions and can have different colors. A comparable spectral reflectance of the building roofs with other objects on the ground can cause incorrect building extraction.

Several investigations have been made to detect building regions using height information. There are two main sources for the height data. One source is LIDAR (LIght Detection And Ranging), which provides very accurate DSM. The majority of solutions propose at first to classify the LIDAR points as whether they belong to the ground, to buildings or to other object classes. ${ }^{9}$ Using ground filters the Digital Terrain Model (DTM) is determined and then subtracted from the DSM. Afterwards, applying height thresholds the remaining nonground points are classified into vegetation and building candidate regions, which are further refined in following

\footnotetext{
*Ksenia.Davydova@dlr.de
} 
steps applying different methods. In order to overcome the possible weakness of horizontal accuracy in LIDAR data some approaches use additionally multispectral data. ${ }^{10,11}$

The other source, which provides elevation information, is stereo imagery. The DSMs are calculated from satellite stereo data using image matching techniques. The quality of satellite images together with the global coverage dramatically increased in the last years, keeping the cost lower as compared to LIDAR data. As a result, DSMs produced by stereo data have become more popular in the recent years. ${ }^{12,13}$ However, one of the drawbacks of using DSMs from stereo satellite images is that they are not as accurate as the LIDAR based DSMs. Generally, building footprint extraction with stereo DSM is quite similar to the methods using LIDAR data. In Ref. 12, 14 the building footprint candidates are generated as following: First, nDSM is generated by subtraction of DTM from DSM. Second, using the NDVI, calculated from given multispectral data, the vegetation is discriminated from the buildings. Afterwords, shape modeling algorithms are used to precisely delineate the buildings. Ref. 15 proposes to extract rectangular building footprints directly from the DSM using Marked Point Processes (MPP). Simple building outlines in vectorial form are extracted from DSM in Ref. 16 using an energy minimization model with the assumption that buildings have rectangular shapes.

Although, many methods were developed in the field of building footprint extraction this problem is still not well solved due to the complexity of buildings in urban areas. In this paper we present a methodology using $\mathrm{MRF}$ and neural networks for automatic building footprint extraction from nDSM and satellite images with a focus on urban areas. A similar approach was used in Ref. 17, where Softmax was applied in the last layer of the neural network, which is actually a pixel-wise decision. The major difference in the presented methodology is the use of MRF for generating the final binary mask, which is a global optimal inference, not a local one.

\section{METHODOLOGY}

\subsection{Problem Formulation}

Similar to Ref. 18 we define our problem as following: Let $I$ be a nDSM image concatenated together with NDVI from given multispectral data and $M$ be a corresponding given building mask. The pixels $M(i, j)$ of the mask could be defined as the following:

$$
M(i, j)= \begin{cases}1 & \text { if }(\mathrm{i}, \mathrm{j}) \text { is building } \\ 0 & \text { if }(\mathrm{i}, \mathrm{j}) \text { is non-building }\end{cases}
$$

We aim to learn how to make the prediction $p(M(i, j) \mid I)$ for each pixel and, applying the MRF energy minimization approach, to generate a binary building mask.

Since the satellite images are huge we tile the images into patches of size $w \times w$ pixels with overlap. It should be mentioned that the size of the patch defined from image is equal to the size of the computed mask patch. Hence, we can rewrite the distribution as:

$$
\left.p\left(\operatorname{Patch}(M)_{w \times w}\right) \mid \operatorname{Patch}(I)_{w \times w}\right)
$$

where Patch(Image $)_{w \times w}$ is an image's patch of size $w \times w$ for both satellite image and true building mask. As a result, the model learns to make predictions for each mask patch $p\left(\operatorname{Patch}(M)_{w \times w}\right)$ given a $p\left(\operatorname{Patch}(I)_{w \times w}\right)$ image patch.

\subsection{Neural Networks and Learning algorithm to detect buildings/non-buildings}

In the first step of the proposed approach for building footprint extraction from DSM and satellite images we model the distribution (1) applying neural networks, which have already been used for several applications in photogrammetry and image analyses. ${ }^{17-19}$ In this work the neural network, functional form is denoted as $f$, is a four-layer perceptron where the first-layer is input, the fourth-layer is output and the rest are hidden-layers. The layers except the first one are non-linear layers with a logistic sigmoid activation function $\sigma(x)$ defined by the formula:

$$
\sigma(x)=\frac{1}{1+e^{-x}}
$$


Regularly, the multilayer perceptron maps the input vector data $x_{i}$ to the output vector data $y_{i}$ through a set of non-linear functions (sigmoid activation function $\sigma(x)$ in our case) with parameters $W$ and $b$ as a weights and biases correspondingly. A single layer neural network can be represented as:

$$
f(\mathbf{x})=\sigma\left(\sum_{i} W_{i} x_{i}+b\right)
$$

Therefore, our network can be written as:

$$
f(\mathbf{x})=\sigma\left(\mathbf{W}_{3}^{\top} \sigma\left(\mathbf{W}_{2}^{\top} \sigma\left(\mathbf{W}_{1}^{\top} \mathbf{x}+\mathbf{b}_{1}\right)+\mathbf{b}_{2}\right)+\mathbf{b}_{3}\right)
$$

where $\mathbf{W}_{\mathbf{i}}$ are the weight matrices and $\mathbf{b}_{\mathbf{i}}$ are the bias vectors for each layer.

The reason of choosing a sigmoid function to model our problem is that we want to be sure the predicted values are between 0 and 1 . It means the closer the value to 1 the higher the probability that the pixel belongs to the desired object. It is very useful for the later binary classification problem. Besides, the derivatives of the sigmoid function are easy to calculate, which leads to a faster training. Moreover, the use of a sigmoid function helps in a simple way to introduce non-linearity to the model. Thereby, when a non-linear function is used, more sophisticated models can be expressed.

In the training process of multilayer neural network weights and biases are at first randomly initialized. During every training iteration the inputs are sent to the network and the model outputs the predictions. The model then learns in a supervised manner comparing the resulted predictions with given true data by minimizing the cross entropy and adjusting the weights and biases with backpropagation algorithm using stochastic gradient descent in small batches. In order to prevent the tendency of neural network from overfitting the training data a "weight decay" (namely L2 regularization) is employed. It prevents the weights of growing too large. The cross entropy loss function together with weight decay formally can be written as:

$$
E=-\frac{1}{n} \sum_{x}[y \log \hat{y}+(1-y) \log (1-\hat{y})]+\frac{\lambda}{2 n} \sum_{w} w^{2}
$$

where $\mathrm{n}$ is the size of our training set, $y$ is the corresponding desired output, $\hat{y}$ is a prediction and $\lambda$ is the weight decay coefficient.

To organize the training data the input images are divided into patches. It is also important to integrate as much additional context information as possible into the inputs. Therefore, because trees are usually mixed with buildings, Normalized Difference Vegetation Index (NDVI) computed from the multispectral images is employed
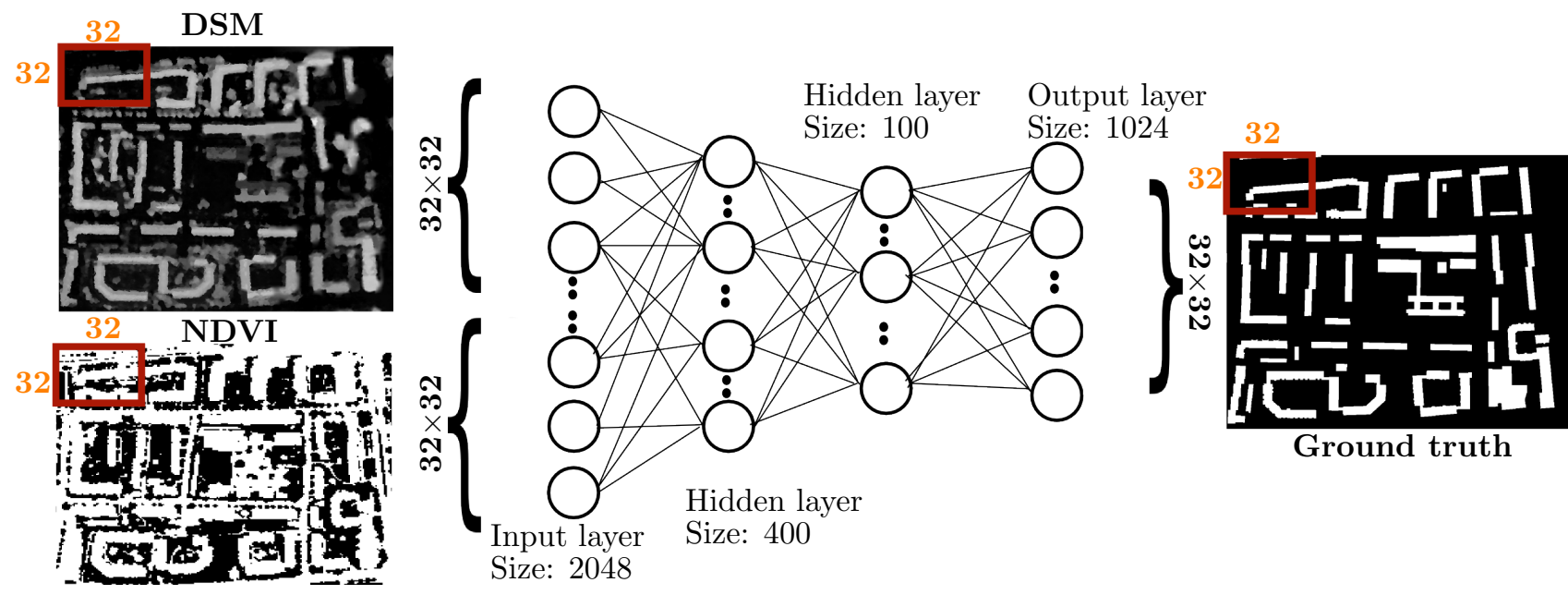
Size: 2048

Figure 1. Neural network organization 
additionally to separate them from buildings. As a result, the neural network is learned on a large set of patches consisting of both DSM and NDVI as inputs and given mask as outputs (see Figure 1).

After training the defined neural network the model is ready to make the predictions on a new unseen data set. Those new data are forwarded though the network and the predicted patches are obtained. After that, the patches are stitched together in the same manner as image tiling in order to generate an image with the same size as the original DSM.

\subsection{MRF energy minimization}

To generate a binary building mask the buildings should be separated from the background. This can be achieved by assigning to each pixel of the image a label. In our case it is a binary form where

$$
L= \begin{cases}0 & \text { background } \\ 1 & \text { building }\end{cases}
$$

Additionally, we need to incorporate spatial correlations into a segmentation process in order to specify the local characteristics of an image. ${ }^{20,21}$ Therefore, the approach for the second step can be formulated as a discrete labeling problem, the solution to which is MRF energy minimization.

MRF is an undirected graphical models which can find a solution to energy minimization via graph cut algorithm, where the minimum cut on a graph corresponds to the minimum of an energy function. ${ }^{22,23}$ It should be mentioned that binary problems can be solved exactly using this approach. We compute minimum cut on a graph by using maximum flow algorithm due to its equivalency to the first as it is declared in the Ford and Fulkerson theorem. ${ }^{23,24}$ This step of the approach is implemented in $\mathrm{C}++$ using the graph cut minimization software prepared by Ref. 25 using the libraries provided by Ref. 26 .

As described above, in the first step the predictions defined by Equation (1) are learned by the chosen neural network and a predicted mask is generated. Mainly, this prediction could be seen as unary terms $\phi_{i}\left(y_{i}, x\right)$ for the MRF energy function as shown in Equation (6).

$$
E=\sum_{i}^{N} \phi_{i}\left(l_{i}, x\right)+\sum_{i \sim j} \psi_{i}\left(l_{i}, l_{j}, x\right)
$$

where $l_{i}$ is the label of node $i, x$ is the given data. The unary terms penalize the solutions inconsistent with the observations. The second potential term of Equation (6) is called pairwise term and it reflects the interactions between labels assign within the neighborhood.

As a result, applying the described methodology we search for the best suitable label for each pixel taking into account spatial correlations between them.

\section{STUDY AREA AND DATASETS}

We performed experiments on datasets consisting of DSM reconstructed from WorldView-2 stereo panchromatic images and NDVI computed from WorldView-2 multi-spectral image. The DSM and panchromatic images have a resolution of 0.5 meters per pixel. By removing a topography information from DSM based on Ref. 27 we obtained a nDSM for the experiment, which contains information only about non-terrain objects (buildings, trees and etc.). For learning the parameters in the neural network a building mask from the municipal city of Munich, Germany is used as a ground truth.

For learning process the training dataset consisting of 39790 samples was generated. This dataset is organized as pairwise dataset, where first element corresponds to the concatenated vector representation of nDSM and NDVI patches both of size $32 \times 32$ (input data for neural network in Figure 1) and the second element, which corresponds to the vector representation of ground truth patches of size $32 \times 32$ (output data for neural network in Figure 1). The overlap between the patches is 24 pixels. To see how well the built neural network can perform, we use the generated on a new area two test datasets, which relate only to the vector representation of nDSM and NDVI patches as inputs to the neural network. 


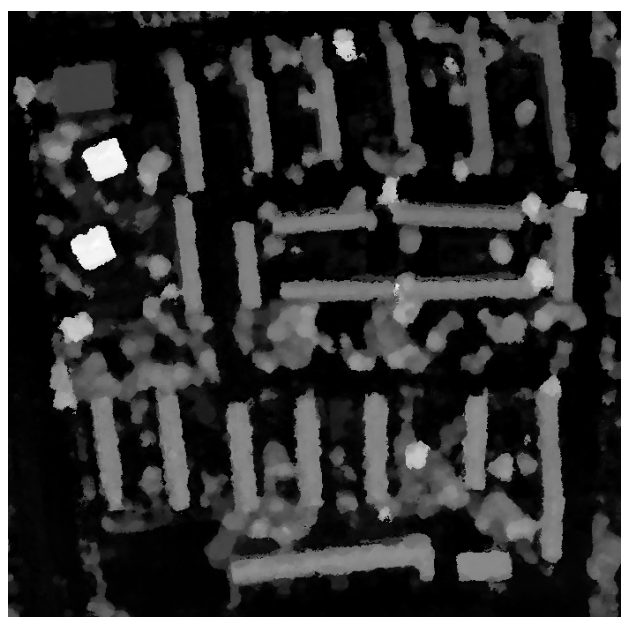

Region 1

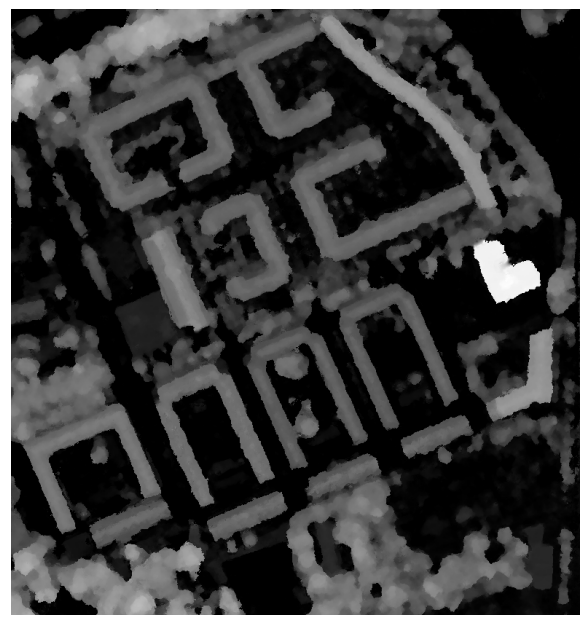

Region 2

Figure 2. Stereo DSMs used for experiment.

The neural network $f$ has 2048 input units, 400 units in the first hidden layer, 100 units in the second hidden layer and 1024 output units. For training the neural networks stochastic gradient descent is used with mini-batches size of 10 . We use a learning rate of 0.0005 and L2 weight decay of 0.0002 . The number of epochs is 3000 .

\section{RESULTS AND DISCUSSIONS}

\subsection{Qualitative analysis}

In this section the results of each step of the proposed method for building footprint extraction from DSM are demonstrated. In order to learn the model parameters for obtaining the probability building mask we train the neural network as described in Section 2.2. After training the neural network and learning the model's parameters, we present to the system the city's areas which have not been used in the training phase (see Figure 2). The building footprint extraction results of the neural network, which provides building/non-building probabilities for each pixel from nDSM are shown in Figure 3 for the selected test datasets. The higher the probability value of a pixel, the brighter the pixel on image and the higher the confidence that the pixel belongs

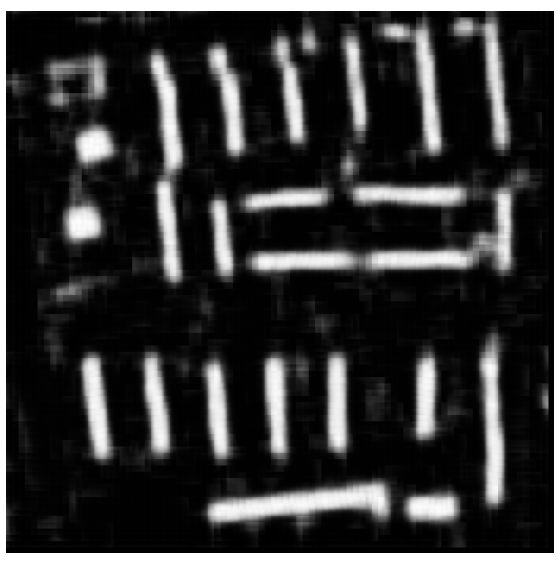

Region 1

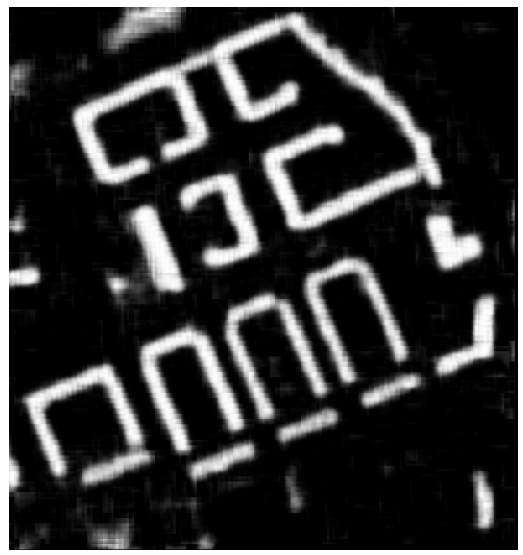

Region 2

Figure 3. Unary energy terms (building/nonbuilding predictions) for each test dataset. 


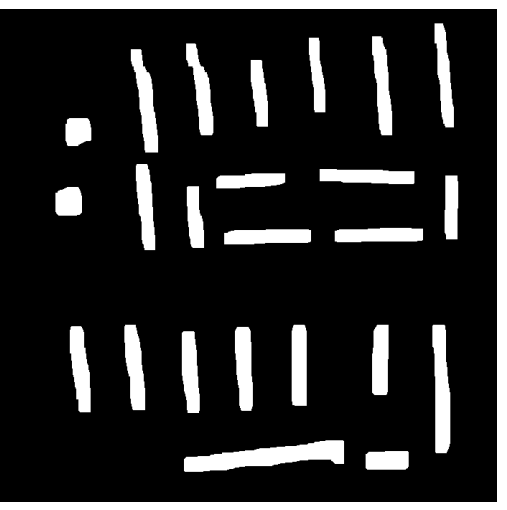

(a) Resulted mask: Region 1

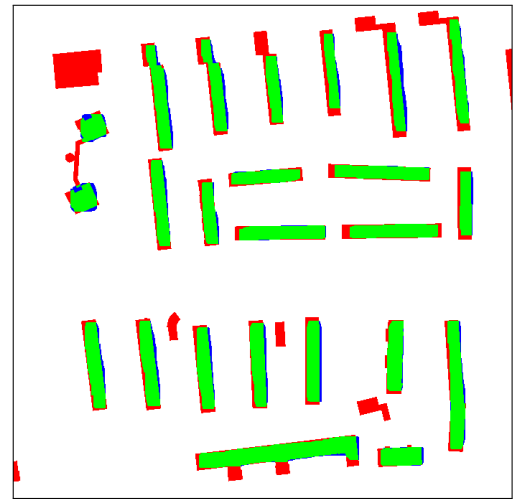

(c) Pixel-based evaluation: Region 1

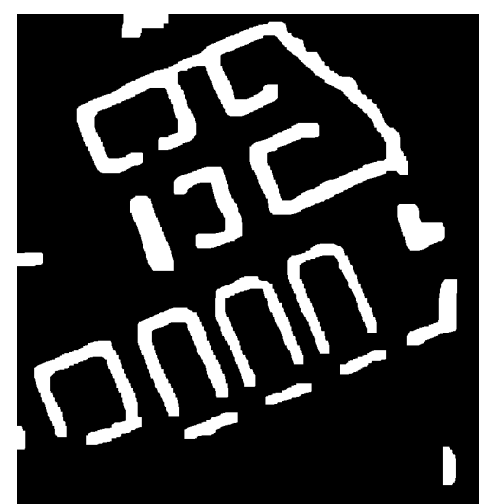

(b) Resulted mask: Region 2

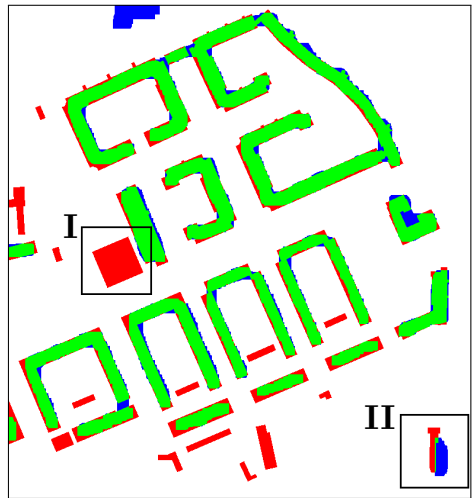

(d) Pixel-based evaluation: Region 2

\section{$\mathrm{TP} \square \mathrm{FN} \square \mathrm{FP}$}

Figure 4. Example of the extracted building footprints from nDSM.

to a building. It can be clearly seen that the model successfully extracts the shapes of building footprints, without any influence of trees.

The aimed binary building masks are obtained for two selected test regions by minimizing the MRF energy function as shown in Figures 4(a) and 4(b). It can be observed that using MRF the complex relationships specifically the building footprint shapes are well modeled. For visualization and better interpretation the extracted building footprints are overlapped with the reference building footprints in Figures 4(c) and 4(d) for both test datasets. As can be seen, the buildings are well separated from the background and they are in most cases very close to the ground truth.

\subsection{Quantitative analysis}

In this paper the building footprint extraction results are evaluated against the reference based on traditional pixel-based and object-based classification accuracy metrics. In the first step a many-to-many correspondence between reference building footprints and the extracted results is registered as the following: if the overlap between a building in reference and an extracted one is at least $50 \%$, then the correspondence is detected. Then the extracted results are categorised as either true positive (TP), true negative (TN), false positive (FP) and false negative (FN). According to classification applications: TP represents the common area of extracted objects and reference objects; TN is an area, which belongs to neither the extracted result nor the reference; FP represents the falsely extracted buildings; FN is the area that belongs to the reference object but not to the extracted 
result. This allows us to introduce the following evaluation metrices for pixel-based evaluation:

$$
\begin{aligned}
\text { Completeness } & =\frac{T P}{T P+F N} \\
\text { Correctness } & =\frac{T P}{T P+F P} \\
\text { Quality } & =\frac{T P}{T P+F N+F P}
\end{aligned}
$$

According to Ref. 28 the description to these metrices can be given as following: The completeness defines the fraction of the building polygon area being correctly detected with respect to the desired building footprint area. The correctness defines the fraction of correctly detected parts of extracted building footprint to the whole extracted building polygon area. The overall quality rate represents a combination of completeness and correctness.

Additionally, object-based shape similarity metrics are calculated as well, which describe how the extracted object is resembling to the referenced one. From the large variety of measures regarding to shape similarities, the Perimeter ratio $r(P)$ and Area ratio $r(A)$ between the extracted building footprint and reference are selected. These ratios are defined as:

$$
\begin{aligned}
& r(P)=\frac{a b s\left(P_{e}-P_{r e f}\right)}{P_{r e f}} \\
& r(A)=\frac{a b s\left(A_{e}-A_{r e f}\right)}{A_{r e f}}
\end{aligned}
$$

where $P_{e}$ and $P_{r e f}$ correspond to perimeter of extracted building and reference, and $A_{e}$ and $A_{r e f}$ are the areas of extracted building and reference, respectively. The closer these ratios to 0 , the more similar are both shapes. It should be noticed that for each presented shape metric, calculated between corresponding buildings, the obtained values are averaged afterwards.

A quantitative analysis has been performed on both selected test datasets to assess the quality of the proposed methodology. For every dataset the pixel-based and object-based metrics were calculated and the results are presented in Table 1. We consider a minimum size of a building as $32 \mathrm{~m}^{2}$ in order to avoid obvious mistakes. From the obtained results we can see that, although the completeness/correctness/quality values are dataset dependent, their values are close to correspondent values among the regions.

Table 1. Pixel-based and object-based automatic building footprint extraction results for both test datasets.

\begin{tabular}{|l|c|c|c|c|c|}
\hline & Completeness & Correctness & Quality & $r(A)$ & $r(P)$ \\
\hline Region 1 & $72 \%$ & $91 \%$ & $67 \%$ & 0.15 & 0.16 \\
\hline Region 2 & $75 \%$ & $89 \%$ & $69 \%$ & 0.13 & 0.078 \\
\hline
\end{tabular}

Most of FN errors (red regions in Figures 4(c) and 4(d)) are due to completely undetected buildings. This can be explained as low sensitivity of our approach to the recognition of low-rise buildings, which are surrounded by higher buildings. Another reason can be the lack of low-rise building or their small amount in the training dataset. As a result, the model did not learn their representations and can not recognize them as buildings. Another reason of undetected buildings is their possible cover with vegetation, grown on the roof. The typical example of such roof is highlighted in black rectangle (I) in Figure 4(d). This region is demonstrated in the multispectral image in Figure 5(a). Some undetected building parts of the obtained results can be caused by noisy DSM data.

Turning to FP errors (blue color in Figures 4(c) and 4(d)) we can conclude that some positively extracted footprints define new built constructions in a city. Because the used ground truth is not up to date, they are not presented on it. However those new constructions can be clearly seen on DSM and pancromatic images. The 


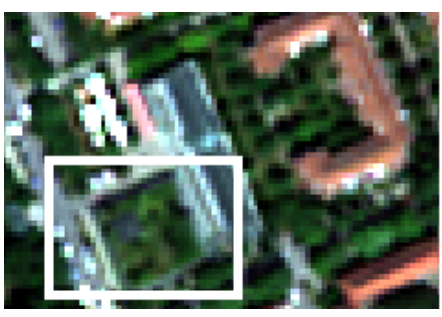

(a) FN detected building:

Roof with vegetation

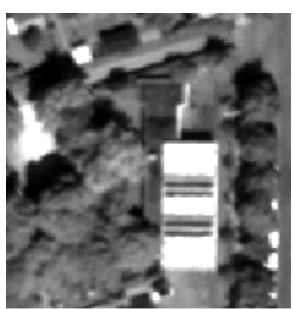

(b) FP detected building: A new construction

Figure 5. Detailed information of the highlighted areas.

detailed view of such FP extracted building (black rectangle (II) in Figure 4(d)) is demonstrated in Figure 5(b)). This is a positive sign that our model can perform also on updating of building footprints.

From the perspective of shape similarity metrics for both regions one can conclude that the outlines of the extracted buildings are close to the reference even for very curved and sophisticated shapes.

\section{CONCLUSION AND FUTURE WORKS}

In this study, a new approach was proposed for automatic building footprint extraction from nDSM using satellite images, neural networks and MRF algorithms. From available multispectral satellite image the NDVI was computed and employed additionally to the nDSM data to separate trees from buildings. Because the images are huge, we tile the DSM and NDVI into patches. In the first step, the four-layer neural network was learned on a large set of patches. Then prediction was performed to calculate the unary terms to be used in the MRF. Further, the energy function was minimized using a max-flow algorithm and a final binary building mask was generated. The major advantage of using the MRF technique, as a post-processing step, is that it gives a global optimal inference, not a local one.

Experimental results have shown that the proposed technique can detect urban and industrial buildings of different shapes with a very high success rate. The undetected buildings on some city's regions can be clarified as a result of low sensitivity of our methodology to the recognition of low-rise buildings due to their lack in the training dataset. As a result, the model did not learn their representations. Additionally, the presence of noise in some parts of DSM causes obvious inaccuracies within the obtained results.

As future implementations we would be interested in trying to improve the available stereo DSM, train the model on a bigger training dataset using parallel programming, build up a generated neural network with MRF as one processing chain and improve the footprint outlines by this.

\section{REFERENCES}

[1] Ahmadi, S., Zoej, M. V., Ebadi, H., Moghaddam, H. A., and Mohammadzadeh, A., "Automatic urban building boundary extraction from high resolution aerial images using an innovative model of active contours," International Journal of Applied Earth Observation and Geoinformation 12(3), 150-157 (2010).

[2] Peng, J., Zhang, D., and Liu, Y., "An improved snake model for building detection from urban aerial images," Pattern Recognition Letters 26(5), 587-595 (2005).

[3] Sohn, G. and Dowman, I., "Extraction of buildings from high resolution satellite data," Automated Extraction of Man-Made Objects from Aerial and Space Images (III). Balkema Publishers, Lisse, 345-355 (2001).

[4] Benediktsson, J. A., Pesaresi, M., and Amason, K., "Classification and feature extraction for remote sensing images from urban areas based on morphological transformations," IEEE Transactions on Geoscience and Remote Sensing 41(9), 1940-1949 (2003).

[5] Ünsalan, C. and Boyer, K. L., "A system to detect houses and residential street networks in multispectral satellite images," Computer Vision and Image Understanding 98(3), 423-461 (2005). 
[6] Shorter, N. and Kasparis, T., "Automatic vegetation identification and building detection from a single nadir aerial image," Remote Sensing 1(4), 731-757 (2009).

[7] Lee, D. S., Shan, J., and Bethel, J. S., "Class-guided building extraction from ikonos imagery," Photogrammetric Engineering \& Remote Sensing 69(2), 143-150 (2003).

[8] San, D. K. and Turker, M., "Building extraction from high resolution satellite images using hough transform," International Archives of the Photogrammetry, Remote Sensing and Spatial Information Science 38(Part 8) (2010).

[9] Lee, D. H., Lee, K. M., and Lee, S. U., "Fusion of lidar and imagery for reliable building extraction," Photogrammetric Engineering 83 Remote Sensing 74(2), 215-225 (2008).

[10] Li, Y. and $\mathrm{Wu}, \mathrm{H} .$, "Adaptive building edge detection by combining lidar data and aerial images," The International Archives of the Photogrammetry, Remote Sensing and Spatial Information Sciences 37, 197202 (2008).

[11] Rottensteiner, F., Trinder, J., Clode, S., and Kubik, K., "Using the dempster-shafer method for the fusion of lidar data and multi-spectral images for building detection," Information fusion 6(4), 283-300 (2005).

[12] San, D. K. and Turker, M., "Automatic building detection and delineation from high resolution space images using model-based approach," in [Proceedings of the ISPRS Workshop on Topographic Mapping from Space], (2006).

[13] Tian, J., Cui, S., and Reinartz, P., "Building change detection based on satellite stereo imagery and digital surface models," IEEE Transactions on Geoscience and Remote Sensing 52(1), 406-417 (2014).

[14] Lu, Y. H., Trunder, J., and Kubik, K., "Automatic building extraction for 3d terrain reconstruction using interpretation techniques," School of Surveying and Spatial Information Systems, University of New South Wales, NSW 2052 (2002).

[15] Brédif, M., Tournaire, O., Vallet, B., and Champion, N., "Extracting polygonal building footprints from digital surface models: a fully-automatic global optimization framework," ISPRS journal of photogrammetry and remote sensing 77, 57-65 (2013).

[16] Ortner, M., Descombes, X., and Zerubia, J., "Building outline extraction from digital elevation models using marked point processes," International Journal of Computer Vision 72(2), 107-132 (2007).

[17] Marmanis, D., Adam, F., Datcu, M., Esch, T., and Stilla, U., "Deep neural networks for above-ground detection in very high spatial resolution digital elevation models," ISPRS Annals of the Photogrammetry, Remote Sensing and Spatial Information Sciences 2(3), 103 (2015).

[18] Mnih, V. and Hinton, G. E., "Learning to detect roads in high-resolution aerial images," in [European Conference on Computer Vision], 210-223, Springer (2010).

[19] Lari, Z. and Ebadi, H., "Automated building extraction from high-resolution satellite imagery using spectral and structural information based on artificial neural networks," in [ISPRS Hannover Workshop], (2007).

[20] Anand, S. S. and Zagorodnov, V., "Retrospective cluster size thresholding for mrf-based detection of activated regions in fmri," in [2006 International Conference on Biomedical and Pharmaceutical Engineering], 44-47, IEEE (2006).

[21] Held, K., Kops, E. R., Krause, B. J., Wells, W. M., Kikinis, R., and Muller-Gartner, H.-W., "Markov random field segmentation of brain $\mathrm{mr}$ images," IEEE transactions on medical imaging 16(6), 878-886 (1997).

[22] Kolmogorov, V. and Rother, C., "Comparison of energy minimization algorithms for highly connected graphs," in [European Conference on Computer Vision], 1-15, Springer (2006).

[23] Boykov, Y., Veksler, O., and Zabih, R., "Fast approximate energy minimization via graph cuts," IEEE Transactions on pattern analysis and machine intelligence 23(11), 1222-1239 (2001).

[24] Ford Jr, L. R. and Fulkerson, D. R., [Flows in networks], Princeton university press (2015).

[25] Kolmogorov, V. and Zabin, R., "What energy functions can be minimized via graph cuts?," IEEE transactions on pattern analysis and machine intelligence 26(2), 147-159 (2004).

[26] Boykov, Y. and Kolmogorov, V., "An experimental comparison of min-cut/max-flow algorithms for energy minimization in vision," IEEE transactions on pattern analysis and machine intelligence 26(9), 1124-1137 (2004). 
[27] Qin, R., Tian, J., and Reinartz, P., "Spatiotemporal inferences for use in building detection using series of very-high-resolution space-borne stereo images," International Journal of Remote Sensing , 1-22 (2015).

[28] Zeng, C., Wang, J., and Lehrbass, B., "An evaluation system for building footprint extraction from remotely sensed data," IEEE Journal of Selected Topics in Applied Earth Observations and Remote Sensing 6(3), 1640-1652 (2013). 